\title{
Reactions of garnet-based solid-state lithium electrolytes with water - a depth-resolved study
}

\author{
Sven Uhlenbruck ${ }^{1-3}$, Christian Dellen ${ }^{1,3}$, Sören Möller ${ }^{1,3}$, Sandra Lobe ${ }^{1,3}$, Chih-Long Tsai ${ }^{1,3}$, Martin \\ Finsterbusch $^{1-3}$, Martin Bram ${ }^{1,3}$, Olivier Guillon ${ }^{1-3}$ \\ ${ }^{1}$ Forschungszentrum Jülich, Institute of Energy and Climate Research, \\ Materials Synthesis and Processing (IEK-1), 52425 Jülich, Germany \\ ${ }^{2}$ Helmholtz Institute Münster: Ionics in Energy Storage (IEK-12), 52425 Jülich, Germany \\ ${ }^{3}$ Jülich Aachen Research Alliance: JARA-Energy, 52425 Jülich, Germany
}

Email corresponding author: s.uhlenbruck@fz-juelich.de

Phone corresponding author: +49-2461-51-5984

\begin{abstract}
Garnet $\mathrm{Li}_{6.4} \mathrm{La}_{3} \mathrm{Zr}_{1.6} \mathrm{Ta}_{0.4} \mathrm{O}_{12}$ thin films prepared by magnetron sputtering were analysed by secondary ion mass spectrometry, nuclear reaction analysis and Rutherford backscattering to identify, localize and quantify the reactions associated with the presence of low amounts of water and carbon dioxide. Samples in a pristine state and after storage in an Argon-filled glove box for months were compared. Both, lithium hydroxide and lithium carbonate were detected, with carbon-containing species and hydrogen-containing having surprisingly different depth profiles.
\end{abstract}

Keywords: battery; thin film; secondary ion mass spectrometry; nuclear reaction analysis; Raman microscopy

\section{Highlights:}

- $\mathrm{Li}_{7-x} \mathrm{La}_{3}(\mathrm{Zr}, \mathrm{Ta})_{2} \mathrm{O}_{12}$ had a distinct reaction zone in the presence of ppm amounts of $\mathrm{H}_{2} \mathrm{O}$

- Spectroscopic methods indicated a change of the crystal lattice

- Secondary ion mass spectrometry revealed a slow migration of $\mathrm{C}$ in the reaction zone

\section{Introduction}

Garnet-based solid-state lithium ion conductors have recently attracted a lot of attention due to their outstanding properties like high ionic conductivity, high stability 
versus lithium ( $\mathrm{Li}$ ) metal, even in the molten state, and high stability against rather high positive potentials $[1,2]$ which might allow battery cells with open circuit voltages significantly higher than about 4.5 Volt. However, this class of materials also exhibits drawbacks like significant chemical reaction with so-called high-voltage cathode active materials during common processing [3], and the sensitivity to moisture. The latter fact has a negative impact on the battery processing and can significantly deteriorate the battery performance.

The reaction of some garnet materials with water and air (incl. moisture and partly also carbon dioxide) is under lively discussion, and there seems to be evidence that the stability crucially depends on details of the composition of the garnet $[1,4,5$, 6]. Many experiments with regard to the reaction of garnets with water have been done under heavy exposure to water $\left(\mathrm{H}_{2} \mathrm{O}\right)$ and carbon dioxide $\left(\mathrm{CO}_{2}\right)$, respectively. $\mathrm{Y}$. Li et al. [7] immersed milled powder in de-ionized water (with $\mathrm{pH} \mathrm{7)}$ ) and found about $21 \%$ of $\mathrm{Li}^{+} / \mathrm{H}^{+}$exchange after 48 hours. C. Ma et al. [5] observed a $\mathrm{Li}^{+} / \mathrm{H}^{+}$ exchange reaction on a time scale of seconds by monitoring the $\mathrm{pH}$ value of an aqueous suspension of garnet $\mathrm{Li}_{7} \mathrm{La}_{3} \mathrm{Zr}_{2} \mathrm{O}_{12}$, and found $\mathrm{a} \mathrm{Li}^{+} / \mathrm{H}^{+}$exchange as high as $\sim 64 \%$ in de-ionized water. W. Xia et al. [8] reported that a certain level of humidity appeared to be required for the reaction of lithium hydroxide $(\mathrm{LiOH})$ and $\mathrm{CO}_{2}$ to lithium carbonate $\left(\mathrm{Li}_{2} \mathrm{CO}_{3}\right)$. Dense pellets were exposed to rather dry $(5 \%$ relative humidity) as well as to humid ( $80 \%$ relative humidity) air for six weeks. The specimens in humid air showed a reaction layer of 100 micrometers while the samples in dry conditions exhibited almost no change. A. Sharafi et al. [9] also stressed the importance of the level of humidity. The group exposed $\mathrm{Li}_{7} \mathrm{La}_{3} \mathrm{Zr}_{2} \mathrm{O}_{12}$ in air with 0.5 and $50 \%$ relative humidity, respectively, for durations of 0 to 240 hours of exposure time. The reaction was much more pronounced with higher humidity. However, they also found by Raman spectroscopy indications for a reaction with low water partial pressure after long exposure time.-W. Xia et al. [6] stored dense pellets in air for three months. Samples prepared in an alumina crucible had slightly increased lattice parameters during storage while samples prepared in a platinum crucible did not. Some reports describe preferred reaction close to grain boundaries $[6,8]$, while reaction layers finally seem to extend over the entire surface $[6,9]$.

The approach by means of extreme conditions is useful to identify general reactions of garnets. In contrast to the publications dealing with the reactions with excessive water, this paper focusses more on sample exposition conditions that are 
closer to a potential handling during processing or operation of solid-state battery cells, like preparing batteries under protective gas or in dry rooms, and operating as pouch cells where the housings may have very high but not perfect gas tightness. Therefore the specimens were stored in inert technical atmospheres with low content of oxygen and water, respectively, i.e. in argon-filled glove boxes with oxygen and water extraction units. Clear indications were found that conditions with water/ air constituent concentrations in the order of magnitude of parts per million may suffice for a proton - lithium ion exchange in garnet $\mathrm{Li}_{6.4} \mathrm{La}_{3} \mathrm{Zr}_{1.6} \mathrm{Ta}_{0.4} \mathrm{O}_{12}$ (nominal composition). The analysis presented in this work is based on depth-resolved methods: nuclear reaction analysis, along with Rutherford backscattering, secondary ion mass spectrometry, and Raman microscopy. These techniques are particularly useful for analysis of lithium-containing compounds compared to methods using $X$ rays: energy/wavelength dispersive $\mathrm{X}$-ray spectroscopy has a very low sensitivity to lithium due to the low atomic number of lithium which directly correlates with low signals. X-ray Photoelectron Spectroscopy (XPS) is limited with regard to the maximum beam penetration/ information depth. A depth-resolved analysis can be performed by sputtering off a few nanometers near the surface after each XPS measurement; however different sputter yields in multi-component systems may make the interpretation more complicated. Secondary ion mass spectrometry has excellently low detection limits for lithium (and also many other elements) and outstanding resolution, however the measured intensities are rather difficult to convert into actual concentrations, and there are also artifacts possible, like so-called "matrix effects", for example certain ions may be removed more easily by the analysis beam in one compound ("matrix") while the same ions are less easily sputtered by the same beam out of a different compound where the ion is bound in a stronger manner. Therefore, as a complementary technique, nuclear reaction analysis was used to quantify the elements in the specimens. In nuclear reaction analysis, a particle beam (for example protons) hits the sample and causes scattering as well as nuclear reactions with the elements. As an example for ${ }^{7} \mathrm{Li}$, protons can be absorbed by the lithium core, which is then instable and quickly decays into $\alpha$ particles of welldefined energy which can be easily detected. A significant advantage is that the energy ranges for reactions with different elements are well separated which allows an unambiguous assignment of the detected charges (equivalent to numbers of particles) and energies to distinct reactions and thus elements. The energy loss of 
the involved particles caused by different interaction processes with the surrounding solid in combination with the cross section of the investigated nuclear reaction also gives an information about the depth resolved elemental concentration.

Raman microscopy is based on photon-phonon interaction and thus provides information about (local) crystal structure. It is sensitive to changes in the chemical bondings and for instance allows measuring the distribution of tetragonal and cubic structures with a spatial resolution in the micrometer range.

\section{Materials and methods}

The nuclear reaction ${ }^{7} \mathrm{Li}(p, \alpha){ }^{4} \mathrm{He}$ was used for ${ }^{7} \mathrm{Li}$ detection. The penetration depth in matter is limited to a few micrometers: the protons have to go into the material and experience a continuous energy loss, react with lithium, and the $\alpha$ particles of the decay process need to get out of the layer without losing all their kinetic energy by scattering processes inside of the specimen. This requires layer thicknesses in the order of micrometers. Coincidently, the storage conditions in a glove box applied in this work lead to thicknesses of additional surface layers of less than one micrometer thus allowing a detection of species from the surface layers as well as from the garnet layer underneath.

Secondary ion mass spectrometry (SIMS) has a typical material erosion rate in the order of magnitude of one micrometer per hour, i.e., SIMS also takes advantage of multilayer set-ups in the range of a few micrometers.

Both techniques benefit from planar, smooth geometries with well-defined planar interfaces. Graded interfacial areas complicate the detection of additional interdiffusion due to signal mixing and artifacts caused by matrix effects in SIMS measurements. Nuclear reaction analysis has less spatial resolution, and planar, sharp interface help to distinguish between adjacent layers. Hence thin films of garnet were prepared by physical vapor deposition on planar substrates. The technical details of the techniques are listed below.

Thin-film preparation of $\mathrm{Li}_{6.4} \mathrm{La}_{3} \mathrm{Zr}_{1.6} \mathrm{Ta}_{0.4} \mathrm{O}_{12}$ : Thin-film coatings of nominally $\mathrm{Li}_{6.4} \mathrm{La}_{3} \mathrm{Zr}_{1.6} \mathrm{Ta}_{0.4} \mathrm{O}_{12}$ (LLZ) were made with a commercial high-vacuum physical vapor deposition (PVD) cluster system CS 800 ES (manufacturer: Von Ardenne Anlagentechnik, Dresden, Germany). Radio frequency (RF) magnetron sputtering 
was used for the preparation of the thin films. FeCrAlY metal alloy sheets (Aluchrom $\mathrm{YHf}^{\circledR}$, material code EN 1.4767) were used as substrates. Details of the target preparation, deposition conditions and subsequent basic analysis of the films by $X$ ray diffraction for phase content and by inductively-coupled plasma optical emission spectrometry can be found in [10].

\section{Sample storing conditions:}

The samples were stored in an argon-filled glove box MB 200 B (manufacturer MBraun, Germany), equipped with water and oxygen sensors and a gas purification system for the removal of oxygen and water. The leakage class was ISO 10648-2, class 1. Argon was taken from a liquid argon gas tank with a rated purity of $99.9999 \%$. The glove box atmosphere contained in the order of magnitude of 1-10 ppm of oxygen and 0.1-1 ppm of water, according to the gas sensors. The pressure inside the glove box was set to 1-1.5 mbar above ambient pressure. The temperature was $20^{\circ} \mathrm{C}$ (laboratory with air condition). The samples were I direct contact to this atmosphere, i.e. they were not specially sealed.

Secondary ion mass spectrometry (SIMS): Time-of-flight (ToF) SIMS analyses were performed with a dual beam setting in non-interlaced mode in a TOF-SIMS IV system (ION-TOF GmbH, Münster, Germany). For the material erosion, a $\mathrm{Cs}^{+}$ion beam was rasterized to produce a sputter crater of $300 \times 300 \mu \mathrm{m}^{2}$. A focused $25 \mathrm{keV} \mathrm{Bi}_{3}{ }^{+}$ion beam was used for the generation of secondary ions for analysis. The analysed area was $82 \times 82 \mu \mathrm{m}^{2}$. The data analysis was done with the Software package SurfaceLab 6.4 (ION-TOF GmbH, Münster, Germany). More experimental details were already published [11]. SIMS is a semi-quantitative analysis tool and allows a comparison between samples with similar matrices (i.e. composition) with high resolution. SIMS data of specimens with different matrices are rather difficult to compare since the quantitative extraction of secondary ions significantly depends on the properties of the specimens. Therefore, Nuclear reaction analysis was used as complementary technique which allows a quantitative assessment of the composition without the need of specific reference measurements for each chemical element.

Nuclear reaction analysis (NRA): A $3 \mathrm{MeV}$ proton beam with $3 \mathrm{~mm}$ in its diameter was generated by a tandetron accelerator. A $50 \mathrm{~mm}^{2}$ passivated implanted planar 
silicon detector with $100 \mu \mathrm{m}$ sensitive thickness and $25 \mathrm{keV}$ FWHM resolution was installed in backscattering geometry at $165^{\circ}$ and at a distance of $100 \mathrm{~mm}$. The proton current was set to $\sim 20 \mathrm{nA}$. The collected charge was about 1 to $80 \mu \mathrm{C}$ for each measurement. Cross-section data from [12] were used for the ${ }^{7} \mathrm{Li}(\mathrm{p}, \alpha)^{4} \mathrm{He}$ reaction, sigmacalc [https://doi.org/10.1016/j.nimb.2015.09.035] for backscattering from 160, $12 \mathrm{C}$ and Rutherford cross-sections for the remaining elements. The results were fitted using the SIMNRA 6.06 software [13]. During the measurement a vacuum pressure of $10^{-7}$ mbar was maintained.

Raman spectroscopy: The measurements were carried out with a Renishaw inVia Qontor Raman microscope using a solid state $532 \mathrm{~nm}$ excitation Laser and an 1800 $\mathrm{I} / \mathrm{mm}$ grating. The collected scans were analysed with the WiRE software (Renishaw). In order to investigate the distribution of chemical components in selected areas of a sample, high-resolution Raman mappings were carried out with a point focus lens. The spectral acquisition time was chosen for sufficiently high resolution and minimal time per spectra ( $1 \mathrm{~s}$ acquisition time, 0.3 micrometer step size). The effective Laser power was set to $2.5 \mathrm{~mW}$, which is $10 \%$ of the maximum power. The reduction of the power was done to avoid sample damage by radiation. The raw data were truncated to $80-1700 \mathrm{~cm}^{-1}$, and a background correction by fitting a third order polynomial function was made for Raman mappings. Noise filter 8 was used for the depth profiles.

\section{Results and discussion}

In Figure 1, a SIMS analysis of a sample of thin-film $\mathrm{Li}_{6.4} \mathrm{La}_{3} \mathrm{Zr}_{1.6} \mathrm{Ta}_{0.4} \mathrm{O}_{12}$ (LLZ) in the pristine state and after ten month storage in a glove box is presented. The positively charged extracted ions are shown. For a better comparison of the two spectra and for a compensation of possible minor differences in the analysis ion beam the intensity of each element is normalized to an area of constant $\mathrm{CsFe}$ intensity within in the respective substrate region. Time $t=0$ corresponds to the start of the analysis of the species sputtered from the surface of the sample exposed to the ambience. Horizontal intensity lines mean a constant concentration of the ions in the directions perpendicular to the plane of the LLZ layer (provided that the ions stem from one specified homogeneous material due to the matrix effect). The concentrations for the 
corresponding ions of $\mathrm{LLZ}$, i.e. $\mathrm{Li}, \mathrm{La}, \mathrm{Zr}$ and $\mathrm{O}$, are apparently rather constant in the direction of analysis which indicates a homogeneous composition of the LLZ layer. Diffusion profiles appear close to the substrate interface, which are associated with the deposition temperature of $700{ }^{\circ} \mathrm{C}$ during the PVD of the LLZ film [10].

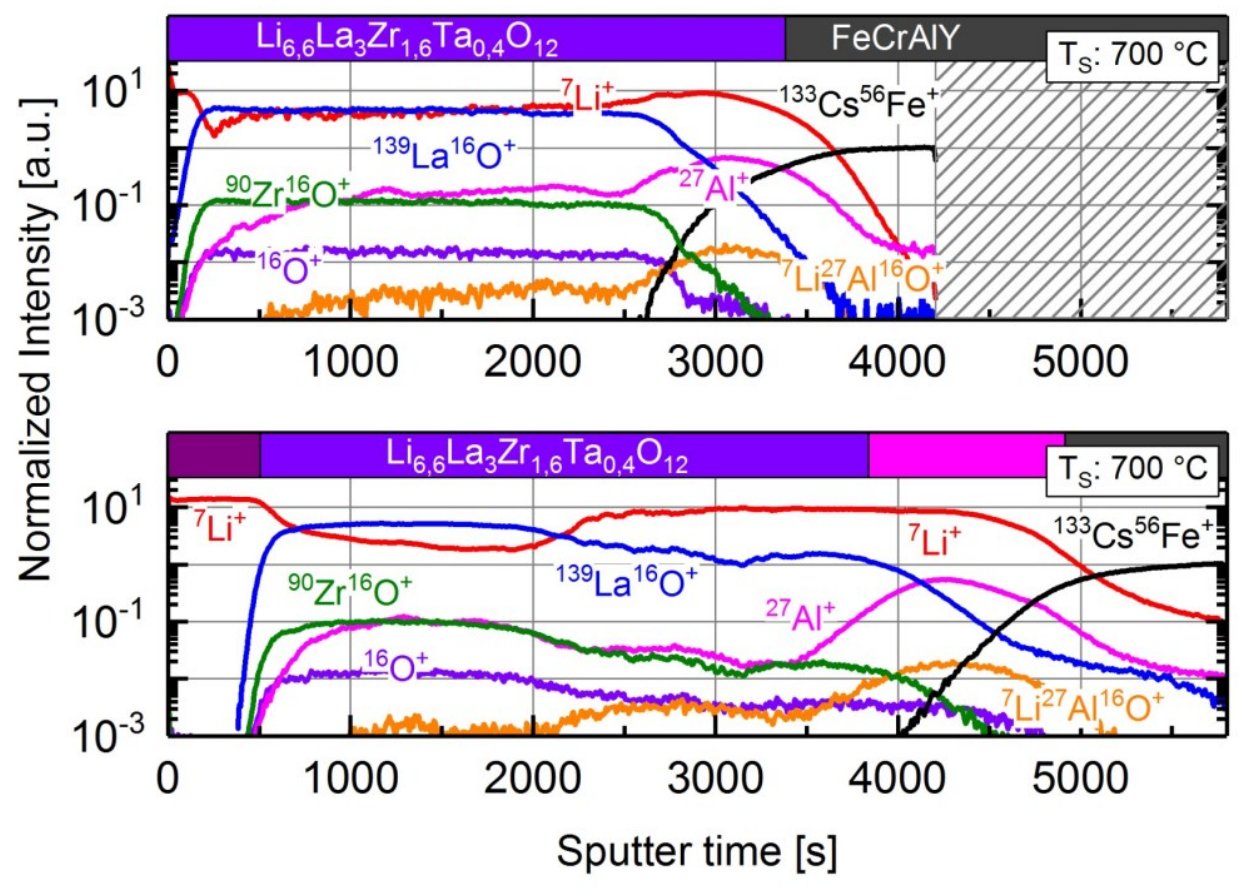

Fig. 1: SIMS measurement (normalized intensity vs. time, positively charged secondary ions) of a thin film of $\mathrm{Li}_{6.4} \mathrm{La}_{3} \mathrm{Zr}_{1.6} \mathrm{Ta}_{0.4} \mathrm{O}_{12}$, top: in pristine state, bottom: after ten months storage in a glove box. The bars at the top of the graphs give an indication of the different layers.

Figure 1 (bottom) shows a SIMS analysis of the sample after long-term storage in an atmosphere with low oxygen and water concentration (see section Materials and methods). New features can be seen in the intensity curves of the different detected ions. Firstly, another layer apparently developed on top of the LLZ layer. The additional layer contains $\mathrm{Li}$, but also hydrogen $(\mathrm{H})$. Since oxygen $(\mathrm{O})$ is present, too, this observation can be an indication for the formation of $\mathrm{LiOH}$, as already described in the literature [7, 8]. Positively charged species with carbon (C) were not detected. Complementary, also the negatively charged ions were recorded from the top layer and analysed (Figure 2). 


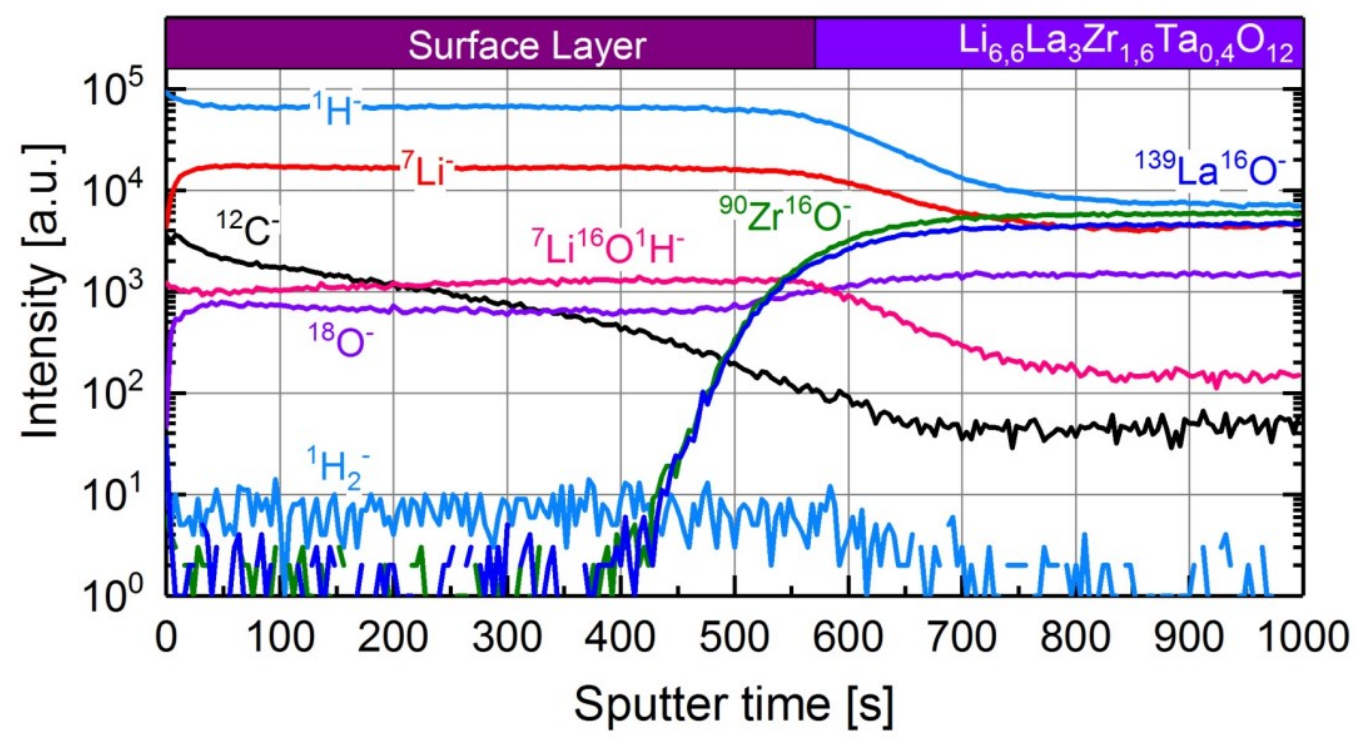

Fig. 2: SIMS analysis of the negatively charged secondary ions of the top layer of the aged LLZ sample.

Carbon could be clearly detected by analysing the negatively charged secondary ions. It is assumed that carbon originates from $\mathrm{CO}_{2} / \mathrm{Li}_{2} \mathrm{CO}_{3}$ [8]. (Note: Carbon in such concentration does not originate from a contamination during processing or handling (like for example oil contamination from high vacuum pumping systems): the intensity of carbon measured by SIMS in samples prepared with the same PVD system and handled and analysed in the same SIMS system were orders of magnitude smaller than the intensity shown in figure 2 [14].

A general question refers to potential origins of $\mathrm{CO}_{2}$. Sensors for carbon dioxide $\left(\mathrm{CO}_{2}\right)$ were not installed in the glove boxes, but it is evident that $\mathrm{CO}_{2}$ might have also been present in the glove box. It can be calculated from the specified leak rate maximum (as an extreme case) given by the manufacturer of the glove boxes that, assuming ideal gas behaviour (i.e. without considering a seal gas effect by the inert gas of the glove box), enough $\mathrm{CO}_{2}$ could intrude into the glove box within a week to convert all lithium of a layer of 0.5 micrometer thickness of the LLZ sample into $\mathrm{Li}_{2} \mathrm{CO}_{3}$. However, it is very likely that the actual leak rate is significantly lower, since lithium metal could be handled in the glove box without any severe reaction. On the other hand, the minimum leak rate in recipients which are sealed with fluorubber ("FKM") is in general in the range of $10^{-8} \mathrm{mbar}$ l/s (the other extreme case), and a calculation revealed that the air intrusion into the glove box would be negligible for 
the aging experiments and results presented in this work. A certain exposition might be possible during mounting the samples in the spectrometer. However, this procedure has been done with the pristine sample, too, and the effect of a second (or third) exposition supposedly cannot explain alone the considerable changes displayed in figures 1 and 2.

The significant differences in the profiles of $\mathrm{Li}, \mathrm{O}$ and $\mathrm{H}$ on the one side, and $\mathrm{C}$ on the other side should be noted. An explanation could be that a rather homogeneous layer containing constant amounts of $\mathrm{Li}, \mathrm{O}$ and $\mathrm{H}$ has been formed, while the $\mathrm{C}$ concentration is apparently highest at the surface of the layer and lowest close to the original LLZ layer. The profile reminds of a non-steady-state diffusion/ concentration profile (note the logarithmic scale on the $y$ axis). Thus, it cannot be excluded that there was first an $\mathrm{H}^{+} / \mathrm{Li}^{+}$exchange in the superficial area of $\mathrm{LLZ}$ thus forming $\mathrm{LiOH}$ as a new layer on the surface of $L L Z$, and afterwards an uptake of $\mathrm{CO}_{2}$ that is somehow limited by the diffusion rate of $\mathrm{CO}_{2}$ or carbonate anions (or by a limited diffusion of the reaction products like excessive water outwards) through the new layer. Such a description is in accordance with a reaction mechanism published by W. Xia et al. [8], who suggest a reaction between $\mathrm{LiOH}$ and $\mathrm{CO}_{2}$ to $\mathrm{Li}_{2} \mathrm{CO}_{3}$ in the presence of water. The $\mathrm{Li}_{2} \mathrm{CO}_{3}$ layer protects to a certain extent the material underneath.

Moreover, new features were found inside of the LLZ layer. In the sputter time range between about 600 and 2500 seconds, a dip in the intensity for $\mathrm{Li}$ is observed. On the first glance, this can be easily understood after the discussion of the reasons for the new top layer on the specimen. The Li-containing top layer requires a source of $\mathrm{Li}$, and it is evident that only the LLZ layer can serve as one. Thus the dip in the intensity can be interpreted by the $\mathrm{H}^{+} / \mathrm{Li}^{+}$exchange in the superficial area of LLZ. However, two more features may be taken into account: (i) The intensity of the detected Li-containing ionic species discloses a quite sharp decrease in the transition zone of the top layer and the LLZ layer, remains more or less constant, and then surprisingly, close to about 2600 seconds sputter time, all detected signals related to $\mathrm{Li}^{+}$suddenly increase again. The question is: Why is there no diffusion profile but such a step? LLZ is known to be a good conductor for lithium ions; its ionic conductivity is according to literature reduced after $\mathrm{H}^{+} / \mathrm{Li}^{+}$exchange: $\mathrm{Y}$. Li et al. [7] describe a reduction of at least one order of magnitude, Y. Wang et al. [15] report a reduction of four orders of magnitude. Nevertheless, the conductivity for $\mathrm{Li}^{+}$is not zero. And supposedly the conductivity for $\mathrm{H}^{+}$after $\mathrm{H}^{+} / \mathrm{Li}^{+}$exchange is also not zero, 
since the exchange is not exclusively limited to an extremely thin surface layer. This deduction means that $\mathrm{H}^{+}$and $\mathrm{Li}^{+}$are assumed to be both mobile in the crystal lattice, and thus either a broad diffusion profile or a constant value for the intensity were expected. Assuming no change in the matrix (i.e. exactly the same host structure (original LLZ crystal lattice) is present in the lithium depleted and in the not lithium depleted garnet (thus excluding a matrix effect) a step-like change in the intensity seems to be incomprehensible. (ii) Not only the intensity lines for Li-containing species show a step, but also the intensity lines of the other elements like for zirconium, oxygen and lanthanum are affected, the intensities in the Li depleted region are higher than in the LLZ region close to the substrate. If the same host structure were present, and thus no matrix effect, the conclusion would be a cumulation of zirconium, lanthanum and oxygen appeared in a distinct area close to the surface of the sample, which is again incomprehensible.

The only hypothesis identified by the authors to explain the data in a reasonable manner is that the $\mathrm{H}^{+} / \mathrm{Li}^{+}$exchange considerably changes the LLZ lattice, thus leading to different intensities due to a matrix effect. The exchange does not change the space group la-3d of cubic LLZ, as pointed out by C. Ma et al. [5] and Y. Li et al. [7]. Nevertheless, changes in the lattice while preserving the space group are reported: W. Xia et al. [6] describe a change in the lattice parameter due to $\mathrm{H}^{+} / \mathrm{Li}^{+}$ exchange, and distortions of $\mathrm{Li}-\mathrm{O}$ octahedra are discussed in the paper of $\mathrm{Y}$. Li et al. [7].

In contrast to SIMS measurements, nuclear reaction analysis is not affected by a matrix effect. Figure 3 shows the result of the analysis of Li in LLZ observed by the nuclear reaction ${ }^{7} \mathrm{Li}(\mathrm{p}, \alpha)^{4} \mathrm{He}$. An additional horizontal scale bar was inserted which was extracted from SEM cross section images of the sample. The scale bar describes the thickness of the layer(s) where Li could be detected. The highest channel number where $\mathrm{Li}$ is detected (around no. 1770) corresponds to the surface of the sample, and the respective energy is the characteristic energy of the $\alpha$ particle: Neither protons nor $\alpha$ particles lose any energy at the sample's surface due to stopping power by matter. 


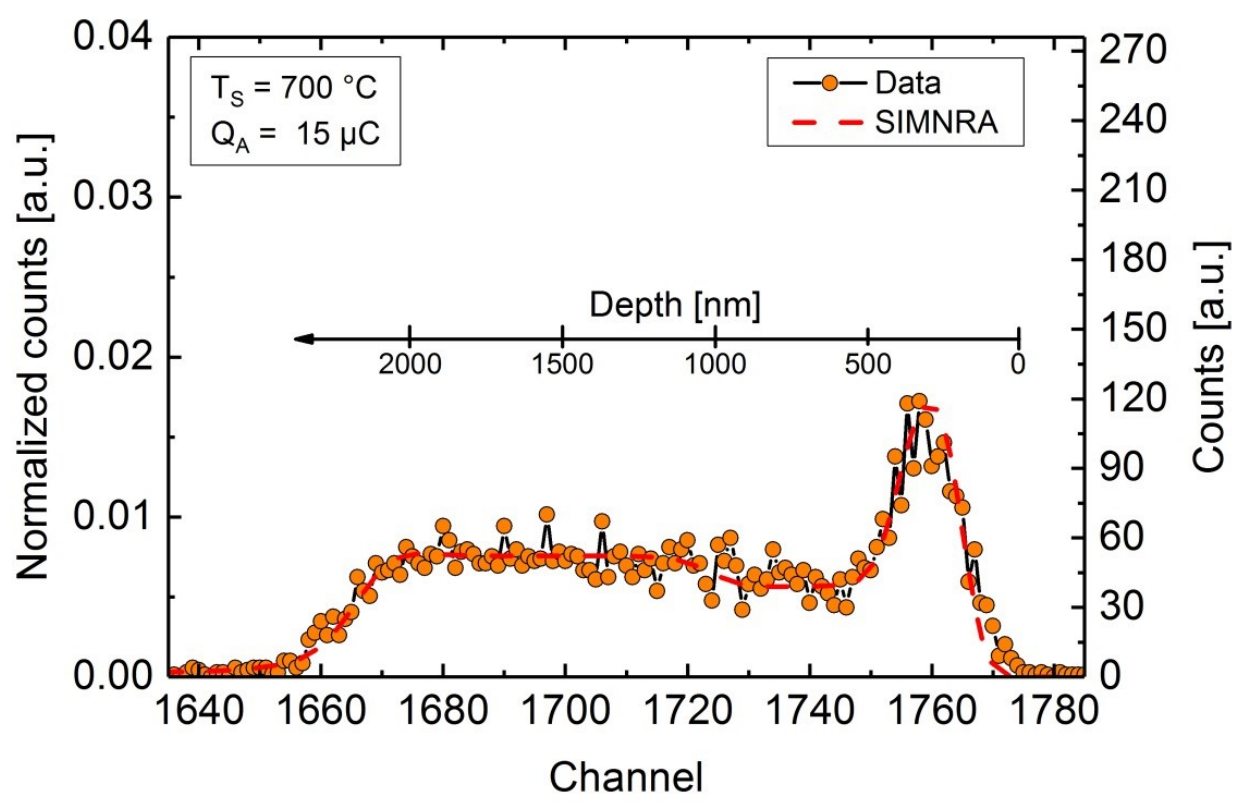

Fig. 3: Energy-selective count rate of $\alpha$ particles corresponding to the ${ }^{7} \mathrm{Li}(p, \alpha)^{4} \mathrm{He}$ nuclear reaction of the aged LLZ sample. An increasing channel number means an increase of the detected $\alpha$ particle's energy. Circles are measured data, the dotted line corresponds to the simulation in SIMNRA.

Higher energies than given by the ${ }^{7} \mathrm{Li}(\mathrm{p}, \alpha)^{4} \mathrm{He}$ reaction are not possible due to energy conservation. Lower energies (equivalent to channels with lower numbers) are correspondingly attributed to $\alpha$ particles that originate from deeper inside the sample. No signal was detected below channel numbers of around 1660. Care was taken that the penetration depths of protons and $\alpha$ particles were higher than the thickness of the layers to be analysed. Thus, the sudden decay of the signal is not due to limitations of the measurement setup (i.e. complete stop of the particles inside of the sample), but due to the absence of lithium below this value. Therefore, the range of channels with $\mathrm{Li} / \alpha$ signal was attributed to the Li-containing layers (LLZ-based layer(s) and the additional top layer).

Figure 3 shows a Li-enriched layer at the surface. which is interpreted as a result of the $\mathrm{H}^{+} / \mathrm{Li}^{+}$exchange and a subsequent formation of $\mathrm{LiOH}$ and $\mathrm{Li}_{2} \mathrm{CO}_{3}$, in accordance to the findings by SIMS (see figures 1 and 2). Towards lower energy channels, i.e. towards the sample substrate, a layer of several of hundreds of nanometers of a thickness with lower Li content can be seen, followed by another layer close to the substrate with a slightly higher Li content. A similar observation was made by SIMS, 
too. The SIMS data revealed an apparent step-like intensity change of the Li signal(s) between these two layers. The limited NRA depth resolution prevents an unambiguous assignment from the NRA data whether there is a step-like change or a more gradual change in Li concentration from one LLZ layer to the other.

Figure 4 shows the Rutherford backscattering (RBS) spectrum of the sample in a range where the signals of $\mathrm{La}, \mathrm{Zr}, \mathrm{Ta}$ and $\mathrm{O}$ as well as $\mathrm{Fe}$ and $\mathrm{Al}$ are located. The signal of $O$ (channel 500 to 550 ) appears rather constant, despite a spike related to the sample surface.

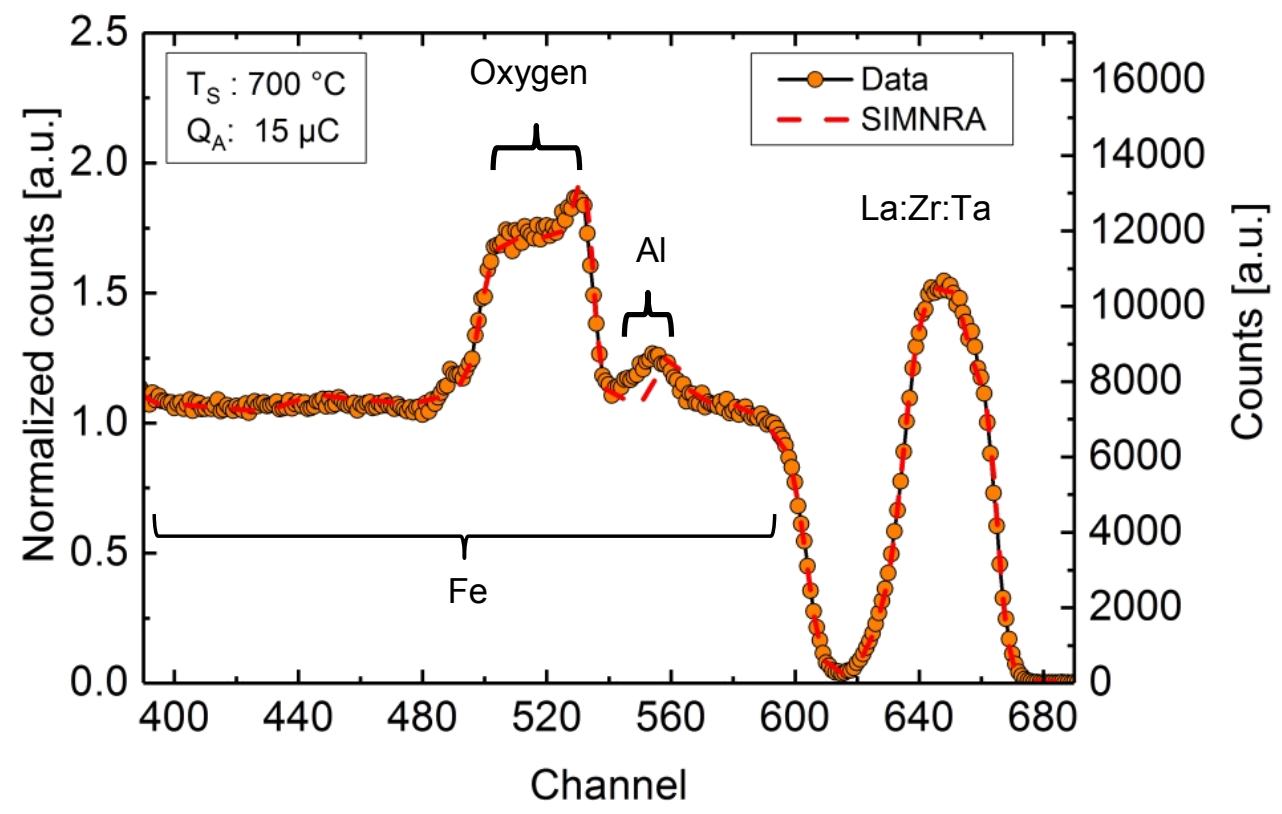

Fig. 4: Rutherford backscattering spectrum of the aged sample. Signals of the LLZ layer and of the substrate are shown. Circles are measured data, the dotted line corresponds to the simulation in SIMNRA.

This spike in oxygen content on the surface of LLZ is assigned to the presence of oxygen-rich compounds, presumably $\mathrm{Li}_{2} \mathrm{CO}_{3}$. The visible amount of the accumulated oxygen at the sample surface appeared to depend on the deposition parameters of the samples: the higher the processing temperature, the more pronounced the oxygen peak [16]. The most self-evident interpretation is that higher deposition temperatures lead to a more intense diffusion of constituents of the substrate into the LLZ layer, and, according to $[1,4,5,6]$, the behavior might depend significantly on the details of the composition of garnet-based materials like LLZ. In a recent paper, Saccoccio et al. published data to LLZ made by pulsed laser deposition at rather low 
temperatures around $300{ }^{\circ} \mathrm{C}$ [17], and the reported SIMS results were described as rather constant, but with the findings of this work, even the slight deviations in the SIMS profiles can be unambiguously interpreted.

The SIMS data exhibited an apparent considerable increase of the intensity of the signals of $\mathrm{La}, \mathrm{Zr}$ and $\mathrm{O}$ in the LLZ layer close to the additional top layer. However, anything similar could not be observed in the RBS data due to the limited depth and mass resolution.

A model consisting of six distinct layers - a top layer, three LLZ layers, an interface layer and the substrate - could best describe the experimental data within the SIMNRA interpretation. The SIMNRA software also allowed a calculation of the elemental composition of the top layer by automated fitting to nominally $\mathrm{Li}_{1.9} \mathrm{C}_{1.1} \mathrm{O}_{3}$. At first sight, this is an intuitively reasonable result since it is close to the expected lithium carbonate $\mathrm{Li}_{2} \mathrm{CO}_{3}$. However, it should be noted that an estimate of the error of the fit for the numbers in the composition yielded $30 \%$ uncertainty of measurement, or even more. Thus it is not possible to distinguish between a pure $\mathrm{Li}_{2} \mathrm{CO}_{3}$ or a mixture with other species, for example physisorbed $\mathrm{CO}_{2}$ or $\mathrm{Li}_{2} \mathrm{O}$. Two potential compounds, namely $\mathrm{LiOH}$ or $\mathrm{H}_{2} \mathrm{O}$, cannot be identified by NRA when using a proton beam, as no backscattering reaction exists, and the corresponding signals of $\mathrm{Li}$ and $\mathrm{O}$ are, if present, superimposed by those of $\mathrm{Li}$ and $\mathrm{O}$ of $\mathrm{Li}_{2} \mathrm{CO}_{3}$. Larraz et al. [18] discussed a reaction of $\mathrm{CO}_{2}$ with $\mathrm{LLZ}$ to form lanthanum oxycarbonates and hydroxycarbonates; the measured NRA data neither support nor contradict to these findings.

The discussion of the SIMS data suggested changes in the crystal lattice, though LLZ in general remains in the same space group la-3d. X-ray diffraction and Raman spectroscopy were used to obtain information of potential changes in the crystal lattice bonds.

X-ray diffraction of a pristine LLZ thin film sample and another LLZ thin film sample after storage in a glove box revealed cubic garnet structure (Figure 5) with sharp reflexes. No indication for tetragonal LLZ or for the competing pyrochlore phase was identified from these data. Slight indications for $\mathrm{Li}_{2} \mathrm{CO}_{3}$ formation were found in the sample with 36 months of storage while $\mathrm{LiOH}$ was apparently not present. 


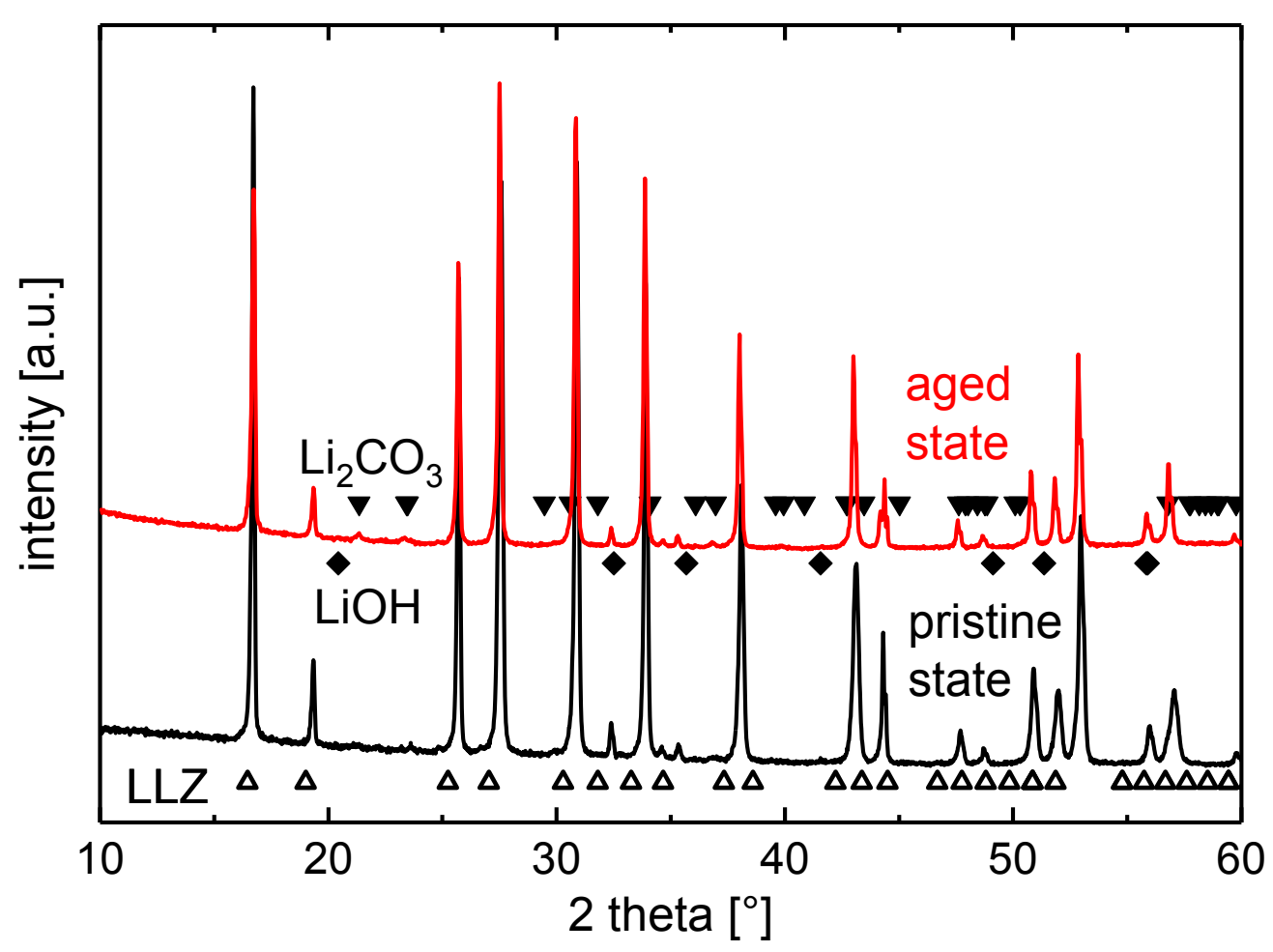

Figure 5: X-ray diffraction pattern of the LLZ thin films in pristine state (bottom) and after 36 months of storage (top) in a glove box, measured in Bragg Brentano geometry. Open triangles show the reflex positions of LLZ according to the Inorganic Crystal Structure Database (ICSD) No. 422259, diamonds: LiOH (ICSD No. 26892), and full triangles: $\mathrm{Li}_{2} \mathrm{CO}_{3}$ (ICSD No. 100324).

Raman microscopy allows a localisation of tetragonal and cubic LLZ phases, respectively, and of $\mathrm{LiOH}$ and $\mathrm{Li}_{2} \mathrm{CO}_{3}$. However, a determination on thin film samples on metal foils were not possible due to the luminescence of the metal substrate which completely covered the Raman signals. Therefore, the Raman measurements shown here were done on ceramic LLZ pellets (details of the preparation can be found in [19]) with rather extreme exposure to clearly identify the spatial distribution of $\mathrm{LiOH}$ and $\mathrm{Li}_{2} \mathrm{CO}_{3}$.

Figure 6 shows Raman spectrums of LLZ samples in the pristine state and after exposure to air/ humidity ("aged sample"). 


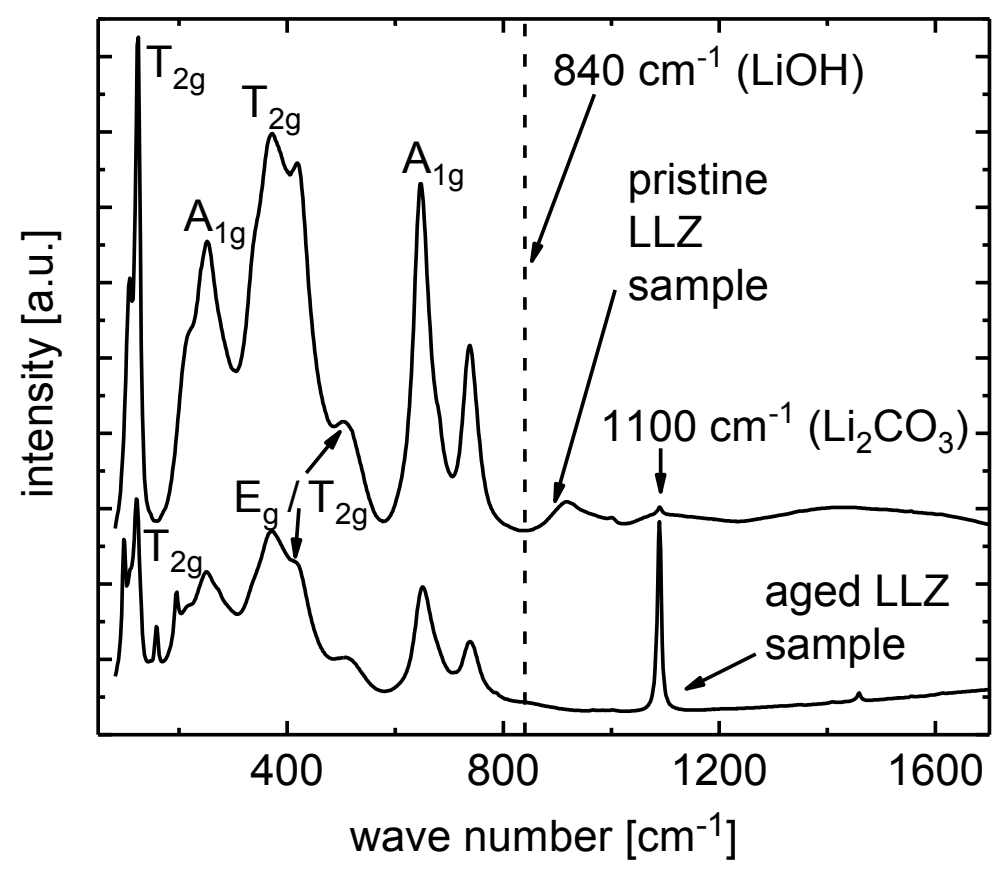

Fig. 6: Averaged Raman spectrum of a mapping of a pristine LLZ bulk sample (sintered ceramic), and after aging in a highly humid atmosphere. The indices of the bands of cubic LLZ are according to [20]. The dotted line at $840 \mathrm{~cm}^{-1}$ shows the potential position of a strong band of $\mathrm{LiOH}$.

Figure 6 reveals a significant change of the Raman bands as a measure of the crystal phase composition in the sample after exposure to air. The pristine sample shows the known pattern of cubic LLZ [20], and no major secondary phases were detected. No evidence for the presence of a tetragonal phase was found [20]. Actually, the cubic phase is expected to be stabilized by the $\mathrm{H}^{+} / \mathrm{Li}^{+}$exchange $[4,5,6$, $7,14]$. After exposition to air, the principal pattern of cubic LLZ is maintained and no tetragonal phase was unambiguously identified. The peak at $1100 \mathrm{~cm}^{-1}$ is attributed to the presence of $\mathrm{Li}_{2} \mathrm{CO}_{3}[6,8]$. The Raman spectrum of $\mathrm{LiOH}$ has a strong band at around $840 \mathrm{~cm}^{-1}$ which does not overlap with the other measured peaks of the pattern of the aged LLZ bulk sample. The aged bulk sample does not contain substantial amounts of $\mathrm{LiOH}$ since no corresponding peak at $840 \mathrm{~cm}^{-1}$ is visible.

Taking LLZ and $\mathrm{Li}_{2} \mathrm{CO}_{3}$ into consideration, the Raman spectrum could be quite well described, however, there are two regions around $275 \mathrm{~cm}^{-1}$ and $770 \mathrm{~cm}^{-1}$ that could not be fitted. The deviations were found to be significant. 
The Empty Modelling simulation within the WIRE software was used to gain more insight into the phases and their distribution. In the framework of the simulation a number of potential phases is provided, and the software develops Raman spectra of phases that could describe the experimental findings by minimizing the variance (least square fit). Figure 7 shows the results for a depth profile of the aged LLZ sample.
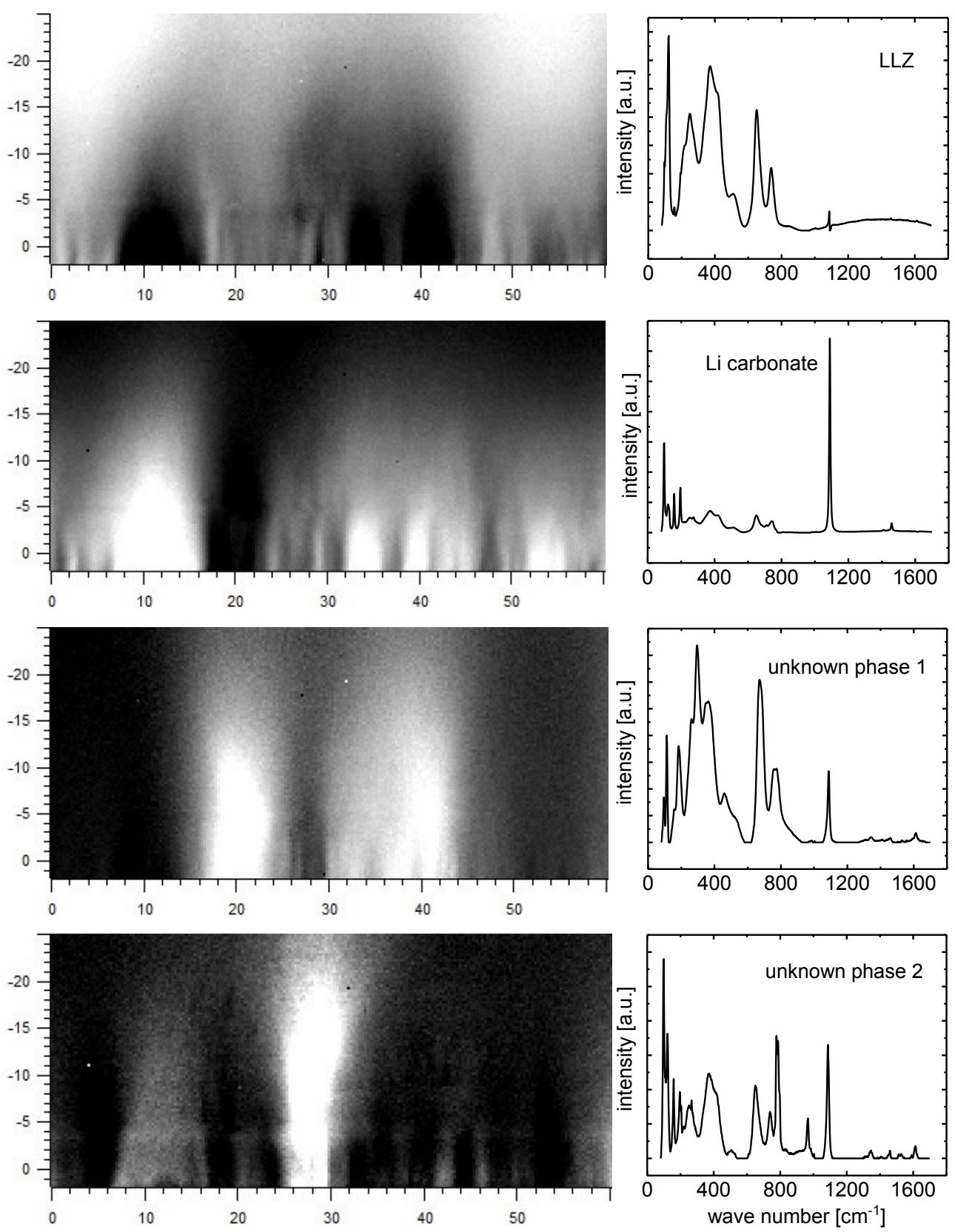

Fig. 7: Raman depth profiles of the aged LLZ sample were simulated with four potential phases (graphs on the right side). Mappings: The distribution of the phases in horizontal direction corresponds to an analysis in lateral direction of the samplethe phase distribution in the sample's depth is shown in y direction. The numbers of the 
axes have the unit micrometers. Each pixel represents one Raman spectrum. The sample surface is at the bottom (close to the $x$ axis).

The best fit was obtained with four phases. The simulated first phase (top) fits very well to the Raman spectrum of $\mathrm{Li}_{2} \mathrm{CO}_{3}$, the second phase to cubic $\mathrm{LLZ}$ (the sharp spike at $1100 \mathrm{~cm}^{-1}$ is interpreted as an artifact by simulation). Two more phases that are necessary to properly describe the entire Raman data could not be identified in this work. No indication for the formation of $\mathrm{La}_{2} \mathrm{O}_{3}$ and $\mathrm{La}_{2} \mathrm{Zr}_{2} \mathrm{O}_{7}$ was found $[21,22]$

\section{Conclusions}

Garnet-based cubic LLZ can react with water and carbon dioxide even at low concentrations of those reactants. The reaction can be best interpreted with a fast initial reaction of $\mathrm{LLZ}$ with moisture, presumably by $\mathrm{H}^{+} / \mathrm{Li}^{+}$exchange. The interaction of LLZ with a carbon containing species might be with slower kinetics but leads to thermodynamically stable phases. SIMS and NRA could identify and localize virtually all elements thus allowing a tracking of the chemical reactions and interdiffusion that happened during processing and handling. Lithium is taken out of a well-defined top layer of LLZ during the reaction, and indications were found that the LLZ crystal lattice is changed by the $\mathrm{H}^{+} / \mathrm{Li}^{+}$exchange while retaining the cubic space group la3d." As another conclusion, the data suggest that the stoichiometry and the processing temperatures/ times of garnets need to be taken into account for a reliable comparison of data of different publications.

\section{Acknowledgement}

Financial support by Helmholtz Gemeinschaft Deutscher Forschungszentren e.V. under grant "Speicher und Vernetzte Infrastrukturen“ and Helmholtz Institute Münster (HI MS), and by Bundesministerium für Bildung und Forschung (Federal ministry of education and research), Germany, under project no. 13N9973, 03X4634C and 03SF0477A is gratefully acknowledged.

\section{References}

[1] R. Murugan, V. Thangadurai, W. Weppner, Fast Lithium lon Conduction in Garnet-Type $\mathrm{Li}_{7} \mathrm{La}_{3} \mathrm{Zr}_{2} \mathrm{O}_{12}$, Angew. Chem. Int. Ed. 46 (2007) 7778-7781 
[2] R. Murugan, V. Thangadurai, W. Weppner, Schnelle Lithiumionenleitung in granatartigem $\mathrm{Li}_{7} \mathrm{La}_{3} \mathrm{Zr}_{2} \mathrm{O}_{12}$, Angew. Chem. Int. Ed. 119 (2007) 7925-7928

[3] L. Miara, A. Windmüller, C.-L. Tsai, W. D. Richards, Q. Ma, S. Uhlenbruck, O. Guillon, G. Ceder, About the compatibility between high voltage spinel cathode materials and solid oxide electrolytes as a function of temperature, ACS Appl. Mater. Interfaces 8 (2016) 26842-26850

[4] F. Gam, C. Galven, A. Bulou, F. Le Berre, M.-P. Crosnier-Lopez, Reinvestigation of the Total $\mathrm{Li}^{+} / \mathrm{H}^{+}$Ion Exchange on the Garnet-Type $\mathrm{Li}_{5} \mathrm{La}_{3} \mathrm{Nb}_{2} \mathrm{O}_{12}$, Inorg. Chem. 53 (2014) 931-934

[5] C. Ma, E. Rangasami, C. Liang, J. Sakamoto, K. L. More, M. Chi, Excellent Stability of a Lithium-lon-Conducting Solid Electrolyte upon Reversible $\mathrm{Li}^{+} / \mathrm{H}^{+}$ Exchange in Aqueous Solutions, Angew. Chem. Int. Ed. 54 (2015) 129-133

[6] W. Xia, B. Xu, H. Duan, Y. Guo, H. Kang, H. Li, H. Liu, lonic Conductivity and Air stability of Al-Doped $\mathrm{Li}_{7} \mathrm{La}_{3} \mathrm{Zr}_{2} \mathrm{O}_{12}$ Sintered in Alumina and Pt Crucibles, ACS Apl. Mater, Interfaces, 8 (2016) 5335-5342

[7] Y. Li, J.-T. Han, S. C. Vogel, C.-A. Wang, The reaction of $\mathrm{Li}_{6.5} \mathrm{La}_{3} Z \mathrm{Zr}_{1.5} \mathrm{Ta}_{0.5} \mathrm{O}_{12}$ with water, Solid State Ionics, 269 (2015) 57-61

[8] W. Xia, B. Xu, H. Duan, X Tang, Y. Guo, H. Kang, H. Li, H. Liu, Reaction mechanisms of lithium garnet pellets in ambient air: The effect of humidity and $\mathrm{CO}_{2}$, J. Am. Ceram. Soc. 100 (2017) 2832-2839

[9] A. Sharafi, S. Yu, M. Naguib, M. Lee, C. Ma, H. M. Meyer, J. Nanda, M. Chi, D. J. Siegel and J. Sakamoto, Impact of air exposure and surface chemistry on Li$\mathrm{Li}_{7} \mathrm{La}_{3} \mathrm{Zr}_{2} \mathrm{O}_{12}$ interfacial resistance, J. Mater. Chem. A, 5 (2017) 13475-13487

[10] S. Lobe, C. Dellen, H.-G. Gehrke, C.-L. Tsai, M. Finsterbusch, D. Sebold, S. Uhlenbruck, O. Guillon, Radio frequency magnetron sputtering of $\mathrm{Li}_{7} \mathrm{La}_{3} \mathrm{Zr}_{2} \mathrm{O}_{12}$ thin 
films for solid-state batteries, J. Power Sources, Journal of Power Sources 307 (2016) 684-689

[11] C. Dellen, H.-G. Gehrke, S. Möller, C.-L. Tsai, U. Breuer, S. Uhlenbruck, O. Guillon, M. Finsterbusch, M. Bram, Time-of-flight secondary ion mass spectrometry study of lithium intercalation process in $\mathrm{LiCoO}_{2}$ thin film, Journal of Power Sources 321 (2016) 241-247

[12] V.Paneta, A. Kafkarkou, M. Kokkoris, A. Lagoyannis, Differential cross-section measurements for the ${ }^{7} \mathrm{Li}\left(\mathrm{p}, \mathrm{p}^{0}\right)^{7} \mathrm{Li},{ }^{7} \mathrm{Li}\left(\mathrm{p}, \mathrm{p}^{1}\right)^{7} \mathrm{Li},{ }^{7} \mathrm{Li}\left(\mathrm{p}, \mathrm{\alpha}^{0}\right)^{4} \mathrm{He},{ }^{19} \mathrm{~F}\left(\mathrm{p}, \mathrm{p}^{0}\right){ }^{19} \mathrm{~F},{ }^{19} \mathrm{~F}\left(\mathrm{p}, \mathrm{\alpha}^{0}\right)^{16} \mathrm{O}$ and ${ }^{19} \mathrm{~F}\left(\mathrm{p}, \mathrm{a}^{1,2}\right)^{16} \mathrm{O}$ reactions , J. Nucl. Instrum. Methods in Physics Res., Sect.B, 288 (2012) 53-59

[13] M. Mayer, SIMNRA, a simulation program for the analysis of NRA, RBS and ERDA, 2016

[14] C. Dellen, Quantitative Analyse der Lithiumverteilung in Kathoden- und Elektrolyt-Dünnschichten für Festkörperbatterien, Energy\&Environment Vol. 363, p. 106, Forschungszentrum Jülich 2017, ISBN 978-3-95806-214-6

[15] Y. Wang, W. Lai, Phase transition in lithium garnet oxide ionic conductors $\mathrm{Li}_{7} \mathrm{La}_{3} \mathrm{Zr}_{2} \mathrm{O}_{12}$ : The role of Ta substitution and $\mathrm{H}_{2} \mathrm{O} / \mathrm{CO}_{2}$ exposure, J. Power Sources 275 (2015) 612-620

[16] C. Dellen, Quantitative Analyse der Lithiumverteilung in Kathoden- und Elektrolyt-Dünnschichten für Festkörperbatterien, Energy\&Environment Vol. 363, p. 122 ff., Forschungszentrum Jülich 2017, ISBN 978-3-95806-214-6

[17] M. Saccoccio, J. Yu, Z. Lu, S. C. T. Kwok, J. Wang, K. K. Yeung, M. M.F. Yuen, $\mathrm{F}$. Ciucci, Low temperature pulsed laser deposition of garnet $\mathrm{Li}_{6.4} \mathrm{La}_{3} \mathrm{Zr}_{1.4} \mathrm{Ta}_{0.6} \mathrm{O}_{12}$ films as all solid-state lithium battery electrolytes, Journal of Power Sources, 365 (2017) $43-52$ 
[18] G. Larraz, A. Orera, M.L. Sanjuán, Cubic phases of garnet-type $\mathrm{Li}_{7} \mathrm{La}_{3} \mathrm{Zr}_{2} \mathrm{O}_{12}$ : the role of hydration, Journal of Materials Chemistry A, 1 (2013) 11419-11428

[19] C.-L. Tsai, V. Roddatis, C. Vinod Chandran, Qianli Ma, Sven Uhlenbruck, Martin Bram, Paul Heitjans, and Olivier Guillon, $\mathrm{Li}_{7} \mathrm{La}_{3} \mathrm{Zr}_{2} \mathrm{O}_{12}$ Interface Modification for $\mathrm{Li}$ Dendrite Prevention, ACS Appl. Mater. Interfaces, 8 (2016) 10617 - 10626

[20] F. Tietz, T. Wegener, M.T. Gerhards, M. Giarola, G. Mariotto, Synthesis and Raman micro-spectroscopy investigation of $\mathrm{Li}_{7} \mathrm{La}_{3} \mathrm{Zr}_{2} \mathrm{O}_{12}$, Solid State lonics 230 (2103) 77-82

[21] J. Cui, G. A. Hope, Raman and Fluorescence Spectroscopy of $\mathrm{CeO}_{2}, \mathrm{Er}_{2} \mathrm{O}_{3}$, $\mathrm{Nd}_{2} \mathrm{O}_{3}, \mathrm{Tm}_{2} \mathrm{O}_{3}, \mathrm{Yb}_{2} \mathrm{O}_{3}, \mathrm{La}_{2} \mathrm{O}_{3}$, and $\mathrm{Tb}_{4} \mathrm{O}_{7}$, Journal of Spectroscopy Volume 2015 (2015) Article ID 940172

[22] M. Pokhrel, M. Alcoutlabi, Y. Mao, Optical and X-ray induced luminescence from $\mathrm{Eu}^{3+}$ doped $\mathrm{La}_{2} \mathrm{Zr}_{2} \mathrm{O}_{7}$ nanoparticles, Journal of Alloys and Compounds 693 (2017) 719-729 